\title{
Systemic hypertension in obstructive sleep apnea
}

\author{
Carolina Lombardi ${ }^{1,2}$, Martino F. Pengo ${ }^{1}$, Gianfranco Parati ${ }^{1,2}$ \\ ${ }^{1}$ Istituto Auxologico Italiano, IRCCS, Dipartimento di Scienze Cardiovascolari, Neurologiche e Metaboliche, S.Luca Hospital, Milan, Italy; \\ ${ }^{2}$ Department of Medicine and Surgery, University of Milano-Bicocca, Milan, Italy \\ Contributions: (I) Conception and design: All authors; (II) Administrative support: G Parati; (III) Provision of study materials or patients: None; \\ (IV) Collection and assembly of data: All authors; (V) Data analysis and interpretation: All authors; (VI) Manuscript writing: All authors; (VII) Final \\ approval of manuscript: All authors. \\ Correspondence to: Carolina Lombardi, MD, PhD. Sleep Disorders Center, Department of Cardiovascular, Neural, and Metabolic Sciences and Sleep \\ Disorders Centre, San Luca Hospital, Istituto Auxologico Italiano IRCCS, Piazzale Brescia 20, 20149 Milan, Italy. Email: c.lombardi@auxologico.it.
}

\begin{abstract}
There is consistent epidemiological evidence that sleep disordered breathing and systemic arterial hypertension are deeply associated, being linked through a bidirectional complex interaction among multiple mechanisms including autonomic nervous system alterations, inflammation, hormonal and hemodynamic components, sleep alterations. However there are several unanswered questions not only from a pathophysiological perspective, but also regarding the effects of obstructive sleep apnea (OSA) treatment on arterial blood pressure values. At present, while many studies have supported the possibility to obtain at least a small blood pressure reduction with OSA treatment, in particular in hypertensive patients, large trials have not clearly confirmed a significant anti-hypertensive effect, nor a beneficial effect of this intervention on cardiovascular endpoints including cardiovascular mortality. Aim of the present review article is to address the relationship between OSA and hypertension in the light of the latest evidence in the field. Moreover we will discuss research topics which need to be investigated in the future, in order to better clarify still pending issues with the aim of obtaining an early diagnosis, a more suitable phenotyping including comorbidities, and better strategies to improve patients' compliance and adherence to treatment.
\end{abstract}

Keywords: Arterial hypertension; sleep apnea; cardiovascular diseases; continuous positive airway pressure (CPAP)

Submitted Aug 08, 2018. Accepted for publication Dec 01, 2018.

doi: $10.21037 /$ jtd.2018.12.57

View this article at: http://dx.doi.org/10.21037/jtd.2018.12.57

\section{Introduction}

The relationship between sleep disordered breathing and systemic arterial hypertension has been extensively studied and there is consistent evidence that these two conditions are deeply associated, being linked through a complex interaction among multiple mechanisms.

After the pioneering papers published in the eighties by the Bologna group (1), which for the first time reported on the association between snoring and blood pressure (BP) elevation, several large population studies, with a crosssectional or a longitudinal design, have been performed on the relation between sleep disordered breathing and $\mathrm{BP}$ changes, whose results have largely confirmed the occurrence of an association between obstructive sleep apnea (OSA) and arterial hypertension.

Overall, the results of cross-sectional observations have shown a prevalence of arterial hypertension in subjects with OSA ranging from $35 \%$ to $80 \%$ (2). When focusing on hypertensive patients, however, OSA prevalence has been reported to be around $40 \%$, increasing to nearly $90 \%$ in patients with resistant hypertension (3). In particular, the association between OSA and hypertension was found to be more evident in young-to middle-aged men $(<50$ years of age). Given their cross sectional nature, however, these studies have been unable to clarify whether OSA and hypertension are simply associated, possibly through concomitant co-morbidities (e.g., obesity), or whether there is a causal link between them, with OSA being responsible for the appearance of new onset hypertension. The evidence 


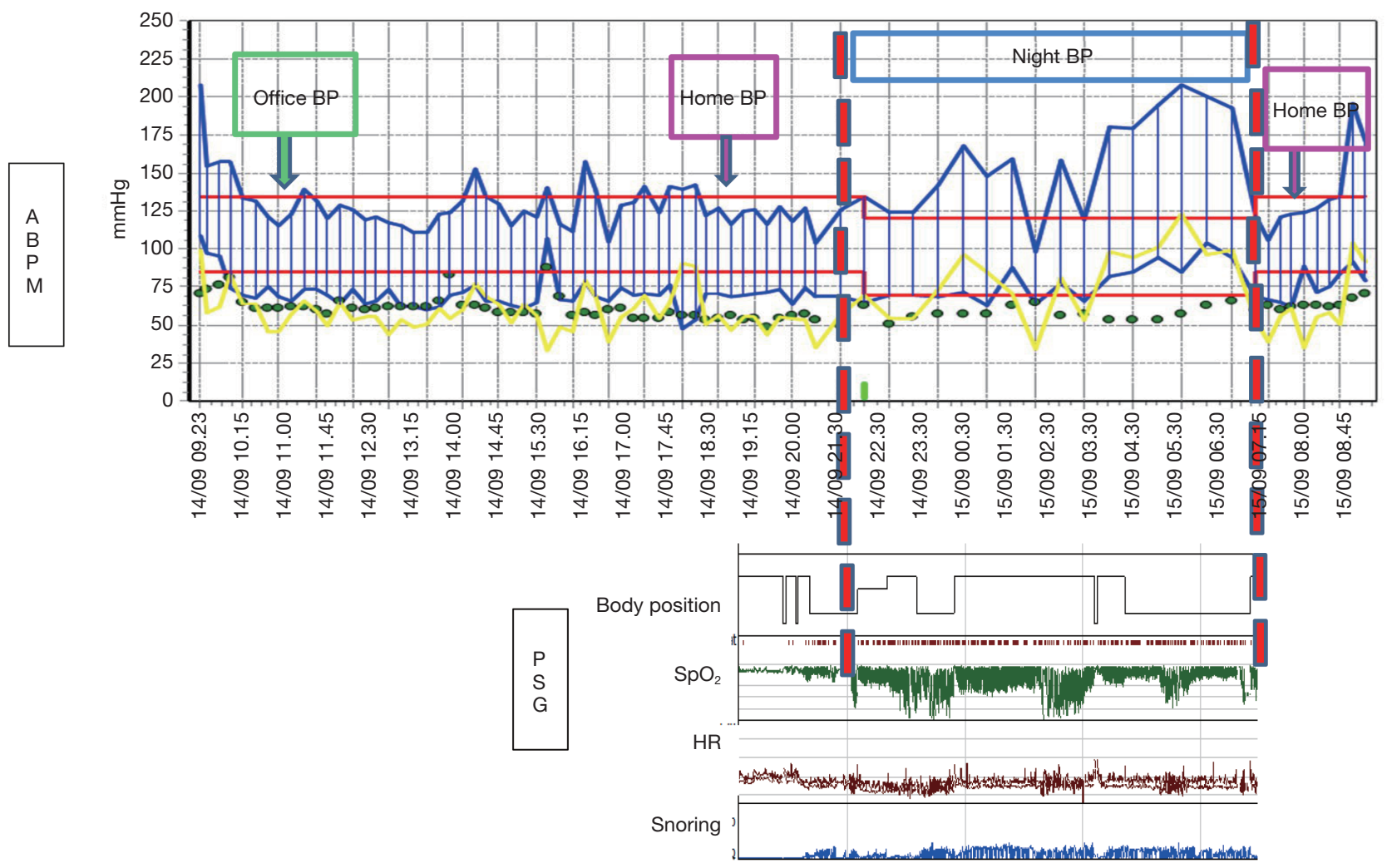

Figure 1 This figure illustrates a 24-hour ambulatory blood pressure profile and polysomnographic tracings obtained in a patient with OSA. The $24 \mathrm{~h}$ BP recording clearly shows an increase of nocturnal of BP values not accompanied by a clear BP elevation at the time of consultation ("office BP", indicating a "masked hypertension condition) nor at the times of home BP self measurements, scheduled in the morning and in the evening according to the ESH home BP guidelines. ABPM, ambulatory blood pressure monitoring; PSG, polysomnography; BP, blood pressure; OSA, obstructive sleep apnea; ESH, European Society of Hypertension.

provided in this regard by available longitudinal surveys is still controversial, with some studies demonstrating a causal link between these two conditions and others being unable to confirm a causal effect of OSA on hypertension after accounting for confounders. The inconsistency among the results of these prospective studies might be partly due to a number of methodological issues, including different selection criteria for patients inclusion at baseline, with a possible recruitment of patients already affected by hypertension at the time of the run-in visit, different follow-up duration, differences in the methodology for OSA evaluation and blood pressure measurement (Figure 1).

Some of these longitudinal studies do indeed support a causal role of OSA on the appearance of hypertension, independently of other potential contributing factors, such as BMI, and have also indicated that the development rate of hypertension is roughly proportional to the severity of OSA, if the latter is not properly treated. In particular, in the Wisconsin Sleep Cohort Study, there was a strong dose dependent relationship between sleep apnea severity and blood pressure: in 709 participants of this study the odds ratios for the development of hypertension at followup were 1.42 (95\% confidence interval, 1.13 to 1.78 ) in patients without sleep disordered breathing and 2.89 (95\% confidence interval, 1.46 to 5.64 ) in patients with mild to severe OSA independently of baseline hypertension status, BMI, neck and waist circumference, age, sex or other potential confounders (4). A causal role of OSA in increasing the incidence of hypertension is also supported by the more recent study published by Marin et al. (5).

Other trials, however, such as the Sleep Heart Health Study (6) and the study by Cano-Pumarega et al. (7), were not able to confirm a causal role of OSA on hypertension development, after accounting for confounders. 
Indeed, there are still many unanswered questions around the link between OSA and hypertension, not only from a pathophysiological perspective, but also regarding the effects of OSA treatment on BP changes. In particular one major issue regards OSA treatment effects in reducing not only the severity of the respiratory disorder, but also the BP levels of these patients and their global cardiovascular risk profile. As a matter of fact, a series of meta-analyses published in the last years have been able to show only a small BP reduction following OSA treatments (8-12) and recent trials have shown that continuous positive airway pressure (CPAP) ventilation, the most effective treatment for obstructive sleep disordered breathing, did not prevent cardiovascular events in a large sample of patients with moderate-to-severe OSA and established cardiovascular disease (13).

On such a background, the purpose of the present review is to address the relationship between OSA and hypertension in the light of the latest evidence in the field.

\section{Pathophysiology of OSA-related hypertension}

OSA is the most prevalent sleep respiratory disorder characterized by recurrent breathing alterations during sleep related to upper airways collapse, either complete (apnea) o partial (hypopnea), associated with persistent breathing efforts (thoracic and abdominal) (14). The end of obstructive events and the resumption of ventilation are associated with brief awakenings (arousals) from sleep, intermittent hypoxia/hypercapnia and blood pressure and heart rate surges.

Several potential mechanisms supporting the relationship between OSA and hypertension have been suggested to play a role, including alterations in autonomic cardiovascular regulation, hormonal and inflammatory changes, changes in intra-thoracic pressure with the related haemodynamic consequences and sleep structure alterations.

\section{Autonomic nervous system alterations}

Seminal studies by the Iowa and Mayo Clinic research groups confirmed previous observations showing that subjects with OSA exhibit blood pressure and heart rate swings at the time of obstructive events during sleep, associated with intermittent surges of sympathetic activity measured with microneurography (15).

One of the relevant and yet pending issues concerning the occurrence of a cause-effect relationship between OSA and hypertension is, however, whether and how much the indisputable occurrence of blood pressure peaks at the time of OSA events at night might extend to the daytime wake period, with a kind of carryover effect of hypertension from sleep to waking hours.

In this regard there is evidence that autonomic alterations, in particular sympathetic overactivity, characterize also daytime hours in OSA patients (16) and that OSA treatment is able to reduce autonomic alterations both during the day and during the night in affected patients $(17,18)$.

CPAP therapy, as well as alternative OSA treatment approaches (i.e., oral appliance devices) have been shown to lower sympathetic activity in OSA subjects supporting the concept that autonomic nervous system changes are the main drive for nocturnal cardiovascular and haemodynamic alterations in sleep disordered breathing $(19,20)$. In the frame of autonomic alterations affecting OSA patients, the increased sympathetic activity typical of OSA is associated with an impaired arterial baroreflex control of the cardiovascular system. There are several reports on the combined occurrence of baroreflex dysfunction and altered cardiovascular variability, during both sleep and wake, in patients with OSA, potentially related also to the excessive daytime sleepiness severity $(21,22)$.

\section{Hormonal and inflammatory changes}

Intermittent hypoxia, particularly if associated with episodes of intermittent re-oxygenation, promotes the production of reactive oxygen species (ROS), activates systemic inflammation, and ultimately impairs endothelial function and patient's metabolism. Kato and colleagues demonstrated that patients with OSA exhibited endothelial dysfunction assessed by blood flow responses to intra-arterial infusions of vasodilators (23).

Moreover there are several studies showing a link between OSA and glucose metabolism alterations (24). There are also intriguing recent reports on the potential role of mineralcorticoid hormones, in particular of aldosterone, in the relationship between OSA and hypertension, Aldosterone is a hormone produced by the outer portion (cortex) of the adrenal gland and is involved in the regulation of water and electrolytes balance in the body. Among patients with resistant hypertension, the occurrence of hyperaldosteronism is significantly associated with the prevalence and severity of OSA (25). In fact, treatment with aldosterone antagonists can reduce not only BP levels, but also the severity of OSA (26). 
One potential mechanism by which hyperaldosteronism contributes to OSA may be an increase in intravascular fluid retention, and the consequent pharyngeal edema. Moreover, aldosterone has been demonstrated to alter endothelial integrity and increase paracellular permeability (27).

The presence of aldosterone receptors also on upper airway smooth muscle cells membranes has been reported, therefore supporting a direct local role played by aldosterone in increasing para-pharyngeal edema, thus favouring OSA (28).

On the other hand, OSA may also cause a hyperaldosteronism condition, by stimulation of the renin-angiotensin-aldosterone system, which is known to be importantly involved in mediating hypertension (29).

Furthermore, the role of extra-renal mineralocorticoid receptors found in heart, brain, vascular smooth muscles, adipocytes, and macrophages is being increasingly recognised as an additional mechanism for blood pressure dis-regulation and vascular remodeling associated with intermittent hypoxia (30). In animal models, aldosterone has been shown to act on central mineralocorticoid receptors to increase brain renin-angiotensin activity and oxidative stress (31). This supports the hypothesis that the aldosterone-induced activation of central mineralocorticoid receptors leads to dysregulation of normal central breathing mechanisms and thus to obstructive sleep apnea.

Finally there is a large amount of studies showing that catecholamines are chronically elevated in OSA, further supporting the role of sympathetic activation in this condition. We have recently described an increase of urinary normetanephrine in patients with OSA and hypertension potentially reduced by CPAP treatment (32).

\section{Hemodynamic mechanisms associated with changes in intrathoracic pressure}

During obstructive apneas, every time there is an upper airway occlusion a series of adverse hemodynamic, chemical and autonomic consequences ensue. The immediate mechanical effect of an obstructive event is the abrupt generation of negative intra-thoracic pressure, which in some otherwise healthy individuals can fall as low as $60 \mathrm{mmHg}$. This phenomenon, affecting cardiac afterload, is typical of OSA. It increases acutely ventricular and atrial wall tension and myocardial oxygen demand (33). These mechanically stressful events repeated over time can contribute to develop left ventricular hypertrophy and atrial remodeling independently of systemic arterial pressure. In this context, the negative effects on myocardial structural changes due to intra-thoracic pressure changes add to those triggered by the recurrent drop of arterial oxygen partial pressure associated with intermittent airway obstructions, further impairing the myocardial oxygen supply/demand ratio. Indeed, hypoxia and hypercapnia are potent, chemoreceptor reflex-mediated, stimuli to the efferent sympathetic nerves discharge and neuronal norepinephrine release.

In summary, cardiac remodeling, common in patients with OSA, is associated with exposures to hypoxemia, catecholamine excess, blood pressure elevation, and intrathoracic pressure swings affecting preload and afterload and left atrial and ventricular transmural pressures.

\section{Sleep structure}

Different sleep stages and changes in sleep structure can affect the cardiovascular, respiratory and endocrine systems also in physiologic conditions (34).

Moreover changes in systemic sleep modulation, when associated with specific risk factors such as obesity, can represent the necessary background for some pathological events, in particular for breathing alterations during sleep.

During REM sleep, in fact, cholinergic-mediated inhibition of the hypoglossal nerve results in the suppression of genioglossus muscle tone, which substantially increases the propensity for upper airway collapse. This can lead to REM-predominant OSA or OSA that becomes more severe during REM sleep. It is well known that REM sleep, compared with non-REM sleep, is associated with greater sympathetic activity and cardiovascular instability in healthy human subjects as well as in patients with OSA. Indeed, OSA in REM sleep, independent of non-REM OSA, is significantly associated with prevalent hypertension and also with an increase in myocardial metabolic demand (35).

The frequent lack of polysomnographic assessment at baseline and at follow up in studies assessing the impact of CPAP on blood pressure may potentially lead to misinterpretation of these studies results on the importance of sleep structure changes. This may occur not only because sleep macro and microstructure cannot be explored, but also because of the possible overlap with other sleep comorbidities such as periodic limb movements.

\section{Bi-directional relationship between OSA and bypertension}

The relationship between OSA and hypertension can be 
bidirectional as intensive blood pressure reductions may influence OSA by stabilizing the upper airways (36). On the other hand, several studies on animals have demonstrated that acute increases in systemic blood pressure cause an inhibitory effect on the EMG of upper airway muscles in cats (37), and anaesthetised dogs (38), thereby facilitating sleep apnea.

Garpestad et al. demonstrated in five awake young men without sleep disorders that mean genioglossal EMG activity decreased with higher mean arterial pressure (39). These findings were not confirmed in a similar study by Wilson et al., in which, in a sample of nine sleeping young adults without sleep apnea, acute blood pressure increases did not alter the average airway resistance (40).

In summary, animal and human data demonstrate that acute increases in blood pressure lead to decrements in upper airway tone and these findings support the possibility that more intensive treatment of nocturnal hypertension might also attenuate sleep apnea severity.

All together these findings represent a robust background which may help explaining the complex pathophysiological link between sleep disordered breathing and arterial hypertension.

However, when exploring the relationship between hypertension and sleep apnea, a number of other factors have to be considered. Among them, the possible finding of different BP phenotypes associated with OSA has to be acknowledged, together with the related impact of different methods used to diagnose hypertension in OSA patients.

\section{Impact of different blood pressure measuring techniques on the relation between OSA and hypertension}

\section{OSA and officelout of office blood pressure values}

Given that blood pressure (BP) can be assessed in different settings it is important to define how BP should be best measured in OSA patients, i.e., whether office $\mathrm{BP}$ readings can be enough or rather whether they have to be accompanied by out of office BP measurements. This is of great methodological importance given that significant differences exist between the two types of measurements. It is well known that office $\mathrm{BP}$ is affected by major methodological limitations, including the intrinsic inaccuracy of the technique, its inability to account for the continuous variations which characterize BP in daily life, and the major influence by the doctor on the BP values measured during consultation, which may be significantly higher than in daily life because of the important impact of the so called "white coat effect", i.e., the BP rise triggered by the emotional impact of the doctor's presence during an office visit. On the other hand, out of office BP measurements better reflect the BP values occurring in daily life, either through self-BP measurements taken at home or by $24 \mathrm{~h}$ ambulatory BP monitoring. The latter offers peculiar advantages in case of OSA patients, insofar it allows to describe the whole $24 \mathrm{~h} \mathrm{BP}$ profile including $\mathrm{BP}$ values at night, by having $\mathrm{BP}$ measurements taken at different times of the day and night and over extended periods of time. Moreover, both home and ambulatory $\mathrm{BP}$ monitoring are free from the alarm reaction triggered by the doctor's visit, i.e., they are free from the white coat effect (41-43).

In untreated patients, the condition characterized by an elevated office BP accompanied by a normal out of office BP is defined as white coat hypertension, while the opposite condition, i.e., a normal office BP accompanied by an elevated out of office $\mathrm{BP}$, is termed "masked hypertension" (MH). While the latter is associated with an increased risk of cardiovascular complications as much as sustained hypertension (in which both office and out of office BP are elevated), the clinical relevance of white coat hypertension is still matter of debate, although data are available that it might not be a fully innocent condition (44).

There are very few studies on the prevalence and the clinical relevance of white coat hypertension in OSA patients, but available data suggests that white coat hypertension (WCH) is a frequent phenomenon in this population and that it could represent a kind of "prehypertension status", which could evolve into sustained hypertension during follow-up $(45,46)$.

It is also important to underline however that in OSA patients also the opposite condition, i.e., masked hypertension, is frequently observed. In such a case, normal office BP values are associated with elevated out of office $\mathrm{BP}$, which most frequently in these patients occur during the night-time, in association with the BP surges induced by repeated obstructive apneas (47).

This condition is indeed quite a common finding in OSA patients and several studies showed that the prevalence of $\mathrm{MH}$ is higher in OSA patients than in the general population $(48,49)$.

The fact that BP increases in these patients are more pronounced during the night, as an acute effect of obstructive 

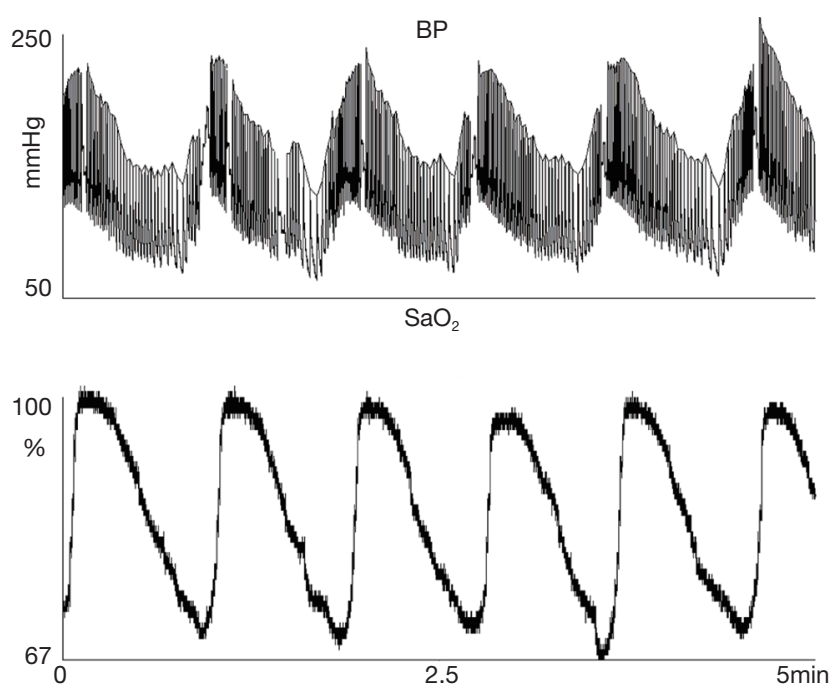

Figure 2 This figure shows a 5 -minute recording of beat by beat blood pressure (BP, continuous finger BP monitoring by a Portapres device, upper panel) and blood oxygen saturation (pulse oximetry, $\mathrm{SpO}_{2}$, lower panel) in a patients-affected by severe OSA. Over the 5 min recording, 6 episodes of obstructive sleep apnea occurred, and the graphs clearly show that OSA episodes were all characterized by the combined occurrence of blood oxygen desaturation (OSA-related intermittent hypoxemia) and blood pressure surges, the latter probably triggered by chemoreflex induced sympathetic activation accompanying each hypoxic event. BP, blood pressure; OSA, obstructive sleep apnea.

events, is also suggested by the typical "non dipping profile" of the $24 \mathrm{~h}$ ambulatory BP recordings of these patients (2) (Figure 1). A recent work has shown that the prevalence of systolic hypertension at night may be significantly higher in children $(n=163$; mean age, $8.2 \pm 3.3$ years; $67 \%$ male) with moderate-to-severe OSA than in those with primary snoring (44.9\% vs. $16.1 \%, \mathrm{P}=0.006)(50)$.

All these data strongly support the importance to perform $24 \mathrm{~h}$ ambulatory BP monitoring in OSA patients both at diagnosis and on treatment during follow-up (2).

\section{Different phenotypes through which the association between OSA and hypertension become manifest}

\section{OSA and blood pressure variability}

Blood pressure is a biological parameter characterized by continuous and marked variations over time also in healthy people, as a consequence of the response of cardiovascular control mechanisms to external perturbations. BP variability may increase, due to a number of intrinsic and/or extrinsic factors, and several studies have shown that an enhanced $\mathrm{BP}$ variability is strongly related to adverse cardiovascular prognosis (42).

BPV consists of fluctuations of blood pressure values across different measurements over a given time interval (51), and is influenced by the interaction among behavioural and environmental stimulations on one side, and the response by humoral and neural control factors, both of central and reflex nature, on the other side (52).

BP fluctuations can be assessed over different time intervals: in the very short term through beat-to-beat BP monitoring, in the short term with a 24-h ambulatory BP monitoring (ABPM), in the mid term, with day by day home BP measurements, and in the long term, through repeated office, home or ambulatory $\mathrm{BP}$ measurements over weeks, months or years, including visit-to-visit BP changes. In particular, short term BPV can be estimated through the standard deviations (SD) of the average systolic and diastolic arterial pressure values over the $24 \mathrm{~h}$, daytime, and nighttime periods, respectively. Due to their different prognostic impact, it is also useful to focus on shorter term BP changes separately from circadian BP fluctuations, by calculating the weighted $24 \mathrm{~h} \mathrm{BP} \mathrm{SD} \mathrm{(average} \mathrm{of} \mathrm{the} \mathrm{daytime} \mathrm{and} \mathrm{the}$ nighttime BP SD separately computed, disregarding the day-night BP difference, and normalised for the duration of daytime and night-time), or the so called average real variability, i.e., the average difference among consecutive $\mathrm{BP}$ readings over a given time window.

$\mathrm{BP}$ variability has been demonstrated, through observational studies and meta-analyses of clinical trials, to be closely related with hypertension mediated target organ damage and to be a significant predictor of mortality, $\mathrm{CV}$ events and stroke (53).

Sleep apnea increases nighttime blood pressure variability in patients with hypertension: in 107 patients BPV measured as the standard deviations of $24 \mathrm{~h}$ and night-time $\mathrm{BP}$ measurements was higher in subjects with OSA $(10.4 \pm 0.7$ versus $8.0 \pm 0.7, \mathrm{P}=0.02)$ compared to controls (54) (Figure 2). However, in a small randomized controlled study, CPAP did not have any effect on short term BPV while 24-h urine norepinephrine levels were associated with both night-time and daytime BP variability (55).

With regards to long term BPV, CPAP determined a reduction in $\mathrm{BPV}$ expressed as the standard deviation of BP measurements taken over three visits repeated 
before and after treatment, but not in absolute values in a cohort of patients with OSA. In this study, mask leak was independently associated with less effective reduction in systolic BPV ( $\mathrm{r}=-0.237, \mathrm{P}=0.048)$ suggesting that also mask related issues may have an impact on haemodynamics and on $\mathrm{BP}$ variations (56).

Withdrawal studies showed that within-visit variability in systolic BP slightly increased in response to CPAP therapy withdrawal, suggesting that the presence of untreated OSA is accompanied by an increased BPV. However, the clinical relevance of this finding has not been fully exploited, despite a possible blunting effect of antihypertensive drugs on BPV (57).

In summary, an increased BPV accompanies OSA, and may contribute to its negative effects on cardiovascular complications. However, the lack of randomized controlled intervention studies make it difficult to recommend the use of CPAP therapy in patients with OSA with the aim of reducing $\mathrm{BPV}$.

\section{OSA and non-dipping BP profile}

Nocturnal BP in healthy people is usually lower when compared with daytime BP, as sleep is known to be associated with a reduction of the sympathetic tone and a prevalence of parasympathetic cardiac modulation. Population based studies including $24 \mathrm{~h}$ ambulatory blood pressure monitoring (ABPM) have demonstrated that patients with a BP drop during the night $<10 \%$ of daytime values (non-dippers) and-even more so-those who exhibit an increase in $\mathrm{BP}$ at night (reverse dippers or risers) show a greater degree of end organ damage, higher risk of stroke, incident heart failure, and increased risk of renal disease progression as compared to those hypertensives with preserved nocturnal BP dipping (58).

The nocturnal dip of BP seen in normal subject is often absent in patients with OSA, in whom such a deranged pattern could be associated with a negative prognostic effect. Studies with ambulatory BP monitoring have indeed shown a more frequent absence of nocturnal BP dipping in OSA patients compared with subjects without OSA. A study by Loredo et al. described an $84 \%$ prevalence of nondipping BP profiles in a sample of untreated patients with mild to severe OSA (59). Loss of BP dipping pattern is mainly attributable to an increase in nocturnal sympathetic activity, which can extend to the daytime, resulting in a permanent increase in sympathetic tone leading to sustained diurnal hypertension.

\section{OSA and resistant bypertension}

OSA has also been found to be strongly associated with resistant hypertension ( $\mathrm{RH})$. $\mathrm{RH}$ is defined as an office BP $\geq 140 / 90 \mathrm{mmHg}$ despite the use of 3 or more antihypertensive agents of different classes (one of which being a diuretic) (60). Patients whose blood pressure is controlled with 4 or more medications are also considered to have resistant hypertension. Patients with resistant hypertension are more likely to experience a cardiovascular event than non-resistant hypertensive patients and OSA is a well-recognized cause of resistant hypertension (61). Among patients with resistant hypertension, the prevalence of OSA is reported to be $70-83 \%(62,63)$.

In this subgroup of hypertensive patients the treatment of OSA can have an enormous impact on both OSA symptoms and blood pressure control. The HIPARCO trial demonstrated that in a sample of 194 patients with resistant hypertension, those randomized to CPAP treatment achieved a better blood pressure control in terms of mean and diastolic $24 \mathrm{~h}$ blood pressure but not in terms of systolic blood pressure (64).

These findings were confirmed by a systematic review and metanalysis where the pooled estimates showed a favourable reduction of $\mathrm{BP}$ with $\mathrm{CPAP}$ treatment in patients with resistant hypertension and OSA (65).

Thus more aggressive screening for OSA in patients with resistant hypertension as well as ensuring treatment compliance in those found to have both resistant hypertension and OSA is mandatory, given that patients with OSA and resistant hypertension represent a peculiar high risk phenotype compared with those OSA patients who do not develop hypertension. Further research and RCTs involving a larger number of study participants, followed over a longer period of time, are warranted to more precisely estimate the level of risk in these patients.

In summary, the different information provided by different methods of BP measurement may become critically important when interpreting not only the results of interventional trials on blood pressure lowering drugs, but also the results of studies focusing on the BP effects of CPAP treatment in OSA patients, where the use of office or of out of office BP monitoring techniques might lead to significantly different conclusions. Indeed, CPAP can determine a reduction in office blood pressure but not in $24 \mathrm{~h}$ blood pressure as shown by Castro-Grattoni and colleagues (66).

Since the time of the first studies demonstrating 
the antihypertensive effect of CPAP in the absence of medication changes or weight loss (67), several randomized trials have been published on this issue, often with conflicting results, however. The Oxford research group demonstrated that CPAP was beneficial in 118 men with OSA as it determined a significant reduction for both systolic and diastolic blood pressure (68).

However, Campos-Rodriguez and colleagues could not replicate the same results as in their cohort of 68 patients with OSA treated with CPAP no significant changes in systolic, diastolic, daytime, or nighttime BP were observed (69).

These results are not surprising giving the vast number of potential confounders, such as different patients characteristics at baseline, use of sub therapeutic CPAP as control group or impact of blood pressure medications, which cannot be properly controlled in a multivariable analysis approach.

\section{Pending issues}

Up to now, current guidelines and recommendations suggest treatment with positive airway pressure to all patients who have been diagnosed with clinically significant OSA (70). Furthermore, in many recommendation papers it is stated that CPAP can be an additional treatment for hypertension when this condition is associated with OSA, without clearly specifying any OSA severity range, nor the blood pressure threshold or the type of BP measurement that should to be used. This uncertainty, is due to the fact that the available randomized controlled studies evaluating the effect of CPAP on $\mathrm{BP}$ are affected by several limitations involving $\mathrm{BP}$ evaluation methods and CPAP treatment implementation (e.g., titration methods, adherence and compliance, time of follow-up). Moreover OSA sleepy patients have often been excluded. For instance, one of the largest trials on sleep apnea and cardiovascular complications did not include patients with an Epworth Sleepiness Scale $>15$, nor those with severe oxygen desaturations $(<80 \%$ for $>10 \%$ of the recording time) (13) This is a crucial issue, because daytime symptoms, such as excessive daytime sleepiness, are associated with the severity of the disease and may express the magnitude of sleep disordered breathing effect also on cardiovascular parameters. Supporting this hypothesis, Ren and colleagues studied OSA patients with multiple sleep latency test and found that excessive daytime sleepiness, when measured objectively, was associated with hypertension (71) and Lombardi et al. showed that in OSA patients objective daytime sleepiness is associated with reduced baroreflex sensitivity during sleep (22).

Furthermore, non-sleepy patients are thought to be at lower risk of cardiovascular disease compared to sleepy patients. In fact, the results of the MOSAIC study support this view, given that minimally symptomatic OSA patients did not show any improvement in daytime ambulatory BP following treatment (72).

Another important aspect which has not been clarified by current recommendations is the degree of reversibility of patients' cardiovascular risk profile following treatment. If we consider cardiovascular risk factors as a continuum in a patient's lifespan, their increasing number or severity leading to progressively greater risk, we can hypothesize that a young patient with no target organ damage is more likely to benefit from CPAP than a patient with established cardiovascular disease. The results of the SAVE trial can be interpreted in such a direction, and because of this, they should not be generalized to all types of OSA patients.

Given these considerations, future studies should not disregard the possibility that a too late start of treatment, when organ damage is already established, might make hypertension resistant not only to drug treatment but also to ventilation by CPAP. This would call for the need of an early diagnosis of OSA and the related hypertension, and for an early start of an effective treatment for both these conditions, with the aim of a more effective reduction in the risk of cardiovascular events.

Additionally, it is important to discuss about CPAP treatment adherence that could play an important role in explaining why some patients with OSA treated with CPAP do not exhibit any improvement with regards to their BP profile. In the SAVE trial, compliance in the CPAP group was limited to about 3.3-hours (mean operating time per night) making the results of the whole trial difficult to interpret. Interestingly, CPAP usage lower than 4 hours per night can potentially leave REM related apneas untreated, given that REM sleep occurs more often towards the end of the night. This is relevant as REM related OSA has been strongly associated with hypertension (35).

Conversely, puzzling observations are available showing that, in patients with good compliance to treatment, other factors often related to CPAP treatment itself might be responsible for a $\mathrm{BP}$ rise rather than favouring control of OSA related hypertension. This apparently paradoxical finding might be explained by the fact that mask air leaks or excessive CPAP pressure can be responsible of recurrent awakenings at night and patient discomfort. It has been 
demonstrated that at increasing CPAP pressure patients experience discomfort and breathlessness (73) together with increased BP levels and enhanced BP variability (74).

Overall, however, the most convincing evidence of the effect of CPAP on BP are from studies on patients with resistant hypertension suggesting that either patients with difficult to control hypertension or with higher $\mathrm{BP}$ values at baseline might exhibit a greater BP drop at follow up. However, predictors of $\mathrm{BP}$ response have not been fully identified and further efforts in this research area are still required, also considering the vast heterogeneity of patients with sleep disordered breathing.

In addition to this, many trials did not consider the additional effect of antihypertensives during CPAP treatment in the enrolled patients. Interestingly, antihypertensives such as Valsartan have been found to be more effective than CPAP on OSA-associated hypertension (75). Thus, the combination of BP-lowering drugs and CPAP can have additive effects on $\mathrm{BP}$ and should be in particular considered in patients with difficult-to-control hypertension. Additional nonpharmacological measures such as lifestyle changes are also likely to have an important role in reducing hypertension related to OSA, but the evidence in this regard is still limited, although encouraging (76). In a randomised trial including 146 patients with obesity and moderate-to-severe OSA, adherence to a regimen of weight loss and CPAP resulted in incremental reductions in BP compared with either intervention alone (77).

Thus, an appropriate management of patients with OSA and hypertension should be multifactorial, including optimisation of type, number and dose of BP lowering drugs, improvement of adherence to treatment for both CPAP and antihypertensive drugs and improvement of life style changes considering the overall cardiovascular risk profile of each patient. The role of other more or less complex interventions, such as surgical procedures, positional therapy, adoption of specific bytes, or selective electrical stimulation of genioglossus nerve, still needs to be defined by ad hoc well performed trials.

\section{Conclusions}

OSA is strongly linked to hypertension by multiple pathophysiological factors, and such a relationship appear to be bidirectional in nature. It is indeed well known that patients with OSA have high probability to be hypertensive and, in turn, it is more probable to find OSA in hypertensive patients.
At present, while many studies have supported the possibility to obtain at least a small $\mathrm{BP}$ reduction with CPAP treatment in OSA patients with hypertension, with the aim of improving their overall cardiovascular profile, large trials have not clearly confirmed a beneficial effect of this intervention on "hard" endpoints such as mortality. This has been recently further emphasized by a systematic review and meta-analysis in which, in contrast with previous studies, meta-regressions identified no associations of CPAP therapy with outcomes for different levels of apnea severity, follow-up duration, or adherence (78).

Further research is therefore needed to better clarify this important issue, also in the light of the many possible confounders listed above.

While waiting for the results of these studies, efforts need nevertheless to be made to improve the time of diagnosis of this condition, the precision of the assessment of the related co-morbidities, including hypertension and diabetes, and patients' compliance and adherence to treatment, which are all important potential determinants of a better clinical outcome.

\section{Acknowledgements}

None.

\section{Footnote}

Conflicts of Interest: The authors have no conflicts of interest to declare.

\section{References}

1. Lugaresi E, Cirignotta F, Coccagna G, et al. Some epidemiological data on snoring and cardiocirculatory disturbances. Sleep 1980;3:221-4.

2. Parati G, Lombardi C, Hedner J, et al. Recommendations for the management of patients with obstructive sleep apnoea and hypertension. Eur Respir J 2013;41:523-38.

3. Parati G, Ochoa JE, Bilo G, et al. Obstructive sleep apnea syndrome as a cause of resistant hypertension. Hypertens Res 2014;37:601-13.

4. Peppard PE, Young T, Palta M, et al. Prospective study of the association between sleep-disordered breathing and hypertension. N Engl J Med 2000;342:1378-84.

5. Marin JM, Agusti A, Villar I, et al. Association between treated and untreated obstructive sleep apnea and risk of hypertension. JAMA 2012;307:2169-76. 
6. O'Connor GT, Caffo B, Newman AB, et al. Prospective study of sleep-disordered breathing and hypertension: the Sleep Heart Health Study. Am J Respir Crit Care Med 2009; 179:1159-64.

7. Cano-Pumarega I, Duran-Cantolla J, Aizpuru F, et al. Obstructive sleep apnea and systemic hypertension: longitudinal study in the general population: the Vitoria Sleep Cohort. Am J Respir Crit Care Med 2011;184:1299-304.

8. Montesi SB, Edwards BA, Malhotra A, et al. The effect of continuous positive airway pressure treatment on blood pressure: a systematic review and meta-analysis of randomized controlled trials. J Clin Sleep Med 2012;8:587-96.

9. Schein AS, Kerkhoff AC, Coronel CC, et al. Continuous positive airway pressure reduces blood pressure in patients with obstructive sleep apnea; a systematic review and meta-analysis with 1000 patients. J Hypertens 2014;32:1762-73.

10. Bratton DJ, Gaisl T, Schlatzer C, et al. Comparison of the effects of continuous positive airway pressure and mandibular advancement devices on sleepiness in patients with obstructive sleep apnoea: a network meta-analysis. Lancet Respir Med 2015;3:869-78.

11. Hu X, Fan J, Chen S, et al. The role of continuous positive airway pressure in blood pressure control for patients with obstructive sleep apnea and hypertension: a metaanalysis of randomized controlled trials. J Clin Hypertens (Greenwich) 2015;17:215-22.

12. Liu L, Cao Q, Guo Z, et al. Continuous Positive Airway Pressure in Patients With Obstructive Sleep Apnea and Resistant Hypertension: A Meta-Analysis of Randomized Controlled Trials. J Clin Hypertens (Greenwich) 2016;18:153-8.

13. McEvoy RD, Antic NA, Heeley E, et al. CPAP for Prevention of Cardiovascular Events in Obstructive Sleep Apnea. N Engl J Med 2016;375:919-31.

14. Garvey JF, Pengo MF, Drakatos P, et al. Epidemiological aspects of obstructive sleep apnea. J Thorac Dis 2015;7:920-9.

15. Narkiewicz K, van de Borne PJ, Cooley RL, et al. Sympathetic activity in obese subjects with and without obstructive sleep apnea. Circulation 1998;98:772-6.

16. Calandra-Buonaura G, Provini F, Guaraldi P, et al. Cardiovascular autonomic dysfunctions and sleep disorders. Sleep Med Rev 2016;26:43-56.

17. Bonsignore MR, Parati G, Insalaco G, et al. Continuous positive airway pressure treatment improves baroreflex control of heart rate during sleep in severe obstructive sleep apnea syndrome. Am J Respir Crit Care Med 2002;166:279-86.

18. Noda A, Nakata S, Koike Y, et al. Continuous positive airway pressure improves daytime baroreflex sensitivity and nitric oxide production in patients with moderate to severe obstructive sleep apnea syndrome. Hypertens Res 2007;30:669-76.

19. Narkiewicz K, Kato M, Phillips BG, et al. Nocturnal continuous positive airway pressure decreases daytime sympathetic traffic in obstructive sleep apnea. Circulation 1999;100:2332-5.

20. Coruzzi P, Gualerzi M, Bernkopf E, et al. Autonomic cardiac modulation in obstructive sleep apnea: effect of an oral jaw-positioning appliance. Chest 2006;130:1362-8.

21. Narkiewicz K, Pesek CA, Kato M, et al. Baroreflex control of sympathetic nerve activity and heart rate in obstructive sleep apnea. Hypertension 1998;32:1039-43.

22. Lombardi C, Parati G, Cortelli P, et al. Daytime sleepiness and neural cardiac modulation in sleep-related breathing disorders. J Sleep Res 2008;17:263-70.

23. Kato M, Roberts-Thomson P, Phillips BG, et al. Impairment of endothelium-dependent vasodilation of resistance vessels in patients with obstructive sleep apnea. Circulation 2000;102:2607-10.

24. Kent BD, Grote L, Bonsignore MR, et al. Sleep apnoea severity independently predicts glycaemic health in nondiabetic subjects: the ESADA study. Eur Respir J 2014;44:130-9.

25. Ke X, Guo W, Peng H, et al. Association of aldosterone excess and apnea-hypopnea index in patients with resistant hypertension. Sci Rep 2017;7:45241.

26. Gaddam K, Pimenta E, Thomas SJ, et al. Spironolactone Reduces Severity of Obstructive Sleep Apnea in Patients with Resistant Hypertension: a Preliminary Report. J Hum Hypertens 2010;24:532-7.

27. Lloberes P, Sampol G, Espinel E, et al. A randomized controlled study of CPAP effect on plasma aldosterone concentration in patients with resistant hypertension and obstructive sleep apnea. J Hypertens 2014;32:1650-7; discussion 1657.

28. Di Murro A, Petramala L, Cotesta D, et al. Reninangiotensin-aldosterone system in patients with sleep apnoea: prevalence of primary aldosteronism. J Renin Angiotensin Aldosterone Syst 2010;11:165-72.

29. Pimenta E, Calhoun DA, Oparil S. Sleep apnea, 
aldosterone, and resistant hypertension. Prog Cardiovasc Dis 2009; 1:371-80.

30. Lam SY, Liu Y, Ng KM, et al. Upregulation of a local renin-angiotensin system in the rat carotid body during chronic intermittent hypoxia. Exp Physiol 2014;99:220-31.

31. Huang BS, Zheng H, Tan J, et al. Regulation of hypothalamic renin-angiotensin system and oxidative stress by aldosterone. Exp Physiol 2011;96:1028-38.

32. Gilardini L, Lombardi C, Redaelli G, et al. Effect of continuous positive airway pressure in hypertensive patients with obstructive sleep apnea and high urinary metanephrines. J Hypertens 2018;36:199-204.

33. Pengo MF, Bonafini S, Fava C, et al. Cardiorespiratory interaction with continuous positive airway pressure. J Thorac Dis 2018;10:S57-70.

34. Marin JM, Carrizo SJ, Vicente E, et al. Long-term cardiovascular outcomes in men with obstructive sleep apnoea-hypopnoea with or without treatment with continuous positive airway pressure: an observational study. Lancet 2005;365:1046-53.

35. Mokhlesi B, Finn LA, Hagen EW, et al. Obstructive sleep apnea during REM sleep and hypertension. results of the Wisconsin Sleep Cohort. Am J Respir Crit Care Med 2014;190:1158-67.

36. Khurshid K, Yabes J, Weiss PM, et al. Effect of Antihypertensive Medications on the Severity of Obstructive Sleep Apnea: A Systematic Review and MetaAnalysis. J Clin Sleep Med 2016;12:1143-51.

37. Mayor AH, Schwartz AR, Rowley JA, et al. Effect of blood pressure changes on air flow dynamics in the upper airway of the decerebrate cat. Anesthesiology 1996;84:128-34.

38. Salamone JA, Strohl KP, Weiner DM, et al. Cranial and phrenic nerve responses to changes in systemic blood pressure. J Appl Physiol Respir Environ Exerc Physiol 1983;55:61-8.

39. Garpestad E, Basner RC, Ringler J, et al. Phenylephrineinduced hypertension acutely decreases genioglossus EMG activity in awake humans. J Appl Physiol (1985) 1992;72:110-5.

40. Wilson CR, Manchanda S, Crabtree D, et al. An induced blood pressure rise does not alter upper airway resistance in sleeping humans. J Appl Physiol (1985) 1998;84:269-76.

41. Parati G, Ochoa JE, Lombardi C, et al. Assessment and interpretation of blood pressure variability in a clinical setting. Blood Press 2013;22:345-54.
42. Parati G, Ochoa JE, Lombardi C, et al. Blood pressure variability: assessment, predictive value, and potential as a therapeutic target. Curr Hypertens Rep 2015;17:537.

43. Parati G, Ochoa JE, Bilo G. Moving Beyond Office Blood Pressure to Achieve a Personalized and More Precise Hypertension Management: Which Way to Go? Hypertension 2017;70:e20-31.

44. Whelton PK, Carey RM, Aronow WS, et al. 2017 ACC/AHA/AAPA/ABC/ACPM/AGS/APhA/ASH/ ASPC/NMA/PCNA Guideline for the Prevention, Detection, Evaluation, and Management of High Blood Pressure in Adults. A Report of the American College of Cardiology/American Heart Association Task Force on Clinical Practice Guidelines. J Am Coll Cardiol 2018;71:e127-248.

45. Garcia-Rio F, Pino JM, Alonso A, et al. White coat hypertension in patients with obstructive sleep apneahypopnea syndrome. Chest 2004;125:817-22.

46. Li L, Guo LZ, Li J, et al. White coat hypertension and obstructive sleep apnea. Sleep Breath 2015;19:1199-203.

47. Parati G, Lombardi C, Hedner J, et al. Position paper on the management of patients with obstructive sleep apnea and hypertension: Joint recommendations by the European Society of Hypertension, by the European Respiratory Society and by the members of European COST (COoperation in Scientific and Technological research) ACTION B26 on Obstructive Sleep Apnea. J Hypertens 2012;30:633-46.

48. Baguet JP, Levy P, Barone-Rochette G, et al. Masked hypertension in obstructive sleep apnea syndrome. J Hypertens 2008;26:885-92.

49. Drager LF, Pedrosa RP, Diniz PM, et al. The effects of continuous positive airway pressure on prehypertension and masked hypertension in men with severe obstructive sleep apnea. Hypertension 2011;57:549-55.

50. Kang KT, Chiu SN, Weng WC, et al. Comparisons of Office and 24-Hour Ambulatory Blood Pressure Monitoring in Children with Obstructive Sleep Apnea. J Pediatr 2017;182:177-83.e2.

51. Parati G, Ochoa JE, Bilo G. Blood pressure variability, cardiovascular risk, and risk for renal disease progression. Curr Hypertens Rep 2012;14:421-31.

52. Parati G, Ochoa JE, Lombardi C, et al. Assessment and management of blood-pressure variability. Nat Rev Cardiol 2013;10:143-55.

53. Pessina AC, Palatini P, Sperti G, et al. Evaluation of hypertension and related target organ damage by 
average day-time blood pressure. Clin Exp Hypertens A 1985;7:267-78.

54. Steinhorst AP, Goncalves SC, Oliveira AT, et al. Influence of sleep apnea severity on blood pressure variability of patients with hypertension. Sleep Breath 2014;18:397-401.

55. Bao X, Nelesen RA, Loredo JS, et al. Blood pressure variability in obstructive sleep apnea: role of sympathetic nervous activity and effect of continuous positive airway pressure. Blood Press Monit 2002;7:301-7.

56. Pengo MF, Ratneswaran C, Berry M, et al. Effect of Continuous Positive Airway Pressure on Blood Pressure Variability in Patients With Obstructive Sleep Apnea. J Clin Hypertens (Greenwich) 2016;18:1180-4.

57. Lettau F, Schwarz EI, Stradling JR, et al. Blood Pressure Variability in Obstructive Sleep Apnoea: Data from 4 Randomised Controlled CPAP Withdrawal Trials. Respiration 2017;93:311-8.

58. Stevens SL, Wood S, Koshiaris C, et al. Blood pressure variability and cardiovascular disease: systematic review and meta-analysis. BMJ 2016;354:i4098.

59. Loredo JS, Ancoli-Israel S, Dimsdale JE. Sleep quality and blood pressure dipping in obstructive sleep apnea. Am J Hypertens 2001;14:887-92.

60. Calhoun DA, Jones D, Textor S, et al. Resistant hypertension: diagnosis, evaluation, and treatment. A scientific statement from the American Heart Association Professional Education Committee of the Council for High Blood Pressure Research. Hypertension 2008;51:1403-19.

61. Somers VK, White DP, Amin R, et al. Sleep apnea and cardiovascular disease: an American Heart Association/ American College of Cardiology Foundation Scientific Statement from the American Heart Association Council for High Blood Pressure Research Professional Education Committee, Council on Clinical Cardiology, Stroke Council, and Council on Cardiovascular Nursing. J Am Coll Cardiol 2008;52:686-717.

62. Gonçalves SC, Martinez D, Gus M, et al. Obstructive sleep apnea and resistant hypertension: a case-control study. Chest 2007;132:1858-62.

63. Logan AG, Perlikowski SM, Mente A, et al. High prevalence of unrecognized sleep apnoea in drug-resistant hypertension. J Hypertens 2001;19:2271-7.

64. Martinez-Garcia MA, Capote F, Campos-Rodriguez $\mathrm{F}$, et al. Effect of CPAP on blood pressure in patients with obstructive sleep apnea and resistant hypertension: the HIPARCO randomized clinical trial. JAMA
2013;310:2407-15.

65. Iftikhar IH, Valentine CW, Bittencourt LR, et al. Effects of continuous positive airway pressure on blood pressure in patients with resistant hypertension and obstructive sleep apnea: a meta-analysis. J Hypertens 2014;32:234150; discussion 2350.

66. Castro-Grattoni AL, Torres G, Martinez-Alonso M, et al. Blood pressure response to CPAP treatment in subjects with obstructive sleep apnoea: the predictive value of 24-h ambulatory blood pressure monitoring. Eur Respir J 2017;50.

67. Logan AG, Tkacova R, Perlikowski SM, et al. Refractory hypertension and sleep apnoea: effect of CPAP on blood pressure and baroreflex. Eur Respir J 2003;21:241-7.

68. Pepperell JCT, Ramdassingh-Dow S, Crosthwaite N, et al. Ambulatory blood pressure after therapeutic and subtherapeutic nasal continuous positive airway pressure for obstructive sleep apnoea: a randomised parallel trial. Lancet 2002;359:204-10.

69. Campos-Rodriguez F, Grilo-Reina A, Perez-Ronchel $J$, et al. Effect of continuous positive airway pressure on ambulatory $\mathrm{BP}$ in patients with sleep apnea and hypertension: a placebo-controlled trial. Chest 2006;129:1459-67.

70. Epstein LJ, Kristo D, Strollo PJ, Jr., et al. Clinical guideline for the evaluation, management and long-term care of obstructive sleep apnea in adults. J Clin Sleep Med 2009;5:263-76.

71. Ren R, Li Y, Zhang J, et al. Obstructive Sleep Apnea With Objective Daytime Sleepiness Is Associated With Hypertension. Hypertension 2016;68:1264-70.

72. Craig SE, Kohler M, Nicoll D, et al. Continuous positive airway pressure improves sleepiness but not calculated vascular risk in patients with minimally symptomatic obstructive sleep apnoea: the MOSAIC randomised controlled trial. Thorax 2012;67:1090-6.

73. Xiao S, Bastianpillai J, Ratneswaran C, et al. Continuous Positive Airway Pressure and Breathlessness in Obese Patients with Obstructive Sleep Apnea: A Pilot Study. Sleep 2016;39:1201-10.

74. Ratneswaran C, Pengo MF, Xiao S, et al. The acute effect of continuous positive airway pressure titration on blood pressure in awake overweight/obese patients with obstructive sleep apnoea. Blood Press 2018;27:206-14.

75. Castro-Grattoni AL, Alvarez-Buve R, Torres M, et al. Intermittent Hypoxia-Induced Cardiovascular Remodeling Is Reversed by Normoxia in a Mouse Model of Sleep 
Apnea. Chest 2016;149:1400-8.

76. Horvath K, Jeitler K, Siering U, et al. Long-term effects of weight-reducing interventions in hypertensive patients: systematic review and meta-analysis. Arch Intern Med 2008;168:571-80.

77. Chirinos JA, Gurubhagavatula I, Teff K, et al. CPAP,

Cite this article as: Lombardi C, Pengo MF, Parati G. Systemic hypertension in obstructive sleep apnea. J Thorac Dis 2018;10(Suppl 34):S4231-S4243. doi: 10.21037/jtd.2018.12.57 weight loss, or both for obstructive sleep apnea. N Engl J Med 2014;370:2265-75.

78. Yu J, Zhou Z, McEvoy RD, et al. Association of Positive Airway Pressure With Cardiovascular Events and Death in Adults With Sleep Apnea: A Systematic Review and Metaanalysis. JAMA 2017;318:156-66. 